\title{
The present-day chemical composition of the SMC from UVES spectra of the sharp-lined, B-type dwarf AV 304
}

\author{
W. R. J. Rolleston ${ }^{1}$, K. Venn ${ }^{2}$, E. Tolstoy ${ }^{3}$, and P. L. Dufton ${ }^{1}$ \\ ${ }^{1}$ Department of Pure \& Applied Physics, The Queen's University of Belfast, BT7 1NN, Northern Ireland, UK \\ 2 University of Minnesota and Macalester College, 1600 Grand Avenue, Saint Paul, MN 55105, USA \\ 3 Kapteyn Institute, University of Groningen, Postbus 800, 9700AV Groningen, The Netherlands
}

Received 29 July 2002 / Accepted 12 November 2002

\begin{abstract}
High-resolution spectroscopic VLT/UVES observations are presented for the B-type main-sequence star, AV 304, in the Small Magellanic Cloud (SMC). These spectra have been analysed using LTE model-atmosphere techniques, to derive stellar atmospheric parameters and chemical compositions. As AV 304 is located within the hydrogen burning main-sequence band, its chemical composition should reflect that of the SMC interstellar medium (ISM). A detailed line-by-line differential analysis has been undertaken relative to a Galactic comparison star. A general metal deficiency for the $\alpha$-process elements $\mathrm{O}$, Si \& S of $-0.43 \pm 0.05$ dex is found for AV 304, with iron having a similar underabundance. Oxygen may be relatively overabundant by $\sim 0.1$ dex and carbon and aluminium underabundant by $\sim 0.2$ dex. A large nitrogen underabundance (of $-1.2 \mathrm{dex}$ relative to hydrogen and -0.7 dex relative to iron) is found. This is interpreted in terms of the CNO bi-cycle having been suppressed in the SMC. Furthermore, the large nitrogen deficiency is in excellent agreement with that found for SMC H II regions. Indeed, this represents a first for stellar astrophysics - confirming the low base-line nitrogen composition of the SMC ISM (viz. $12+\log (\mathrm{N} / \mathrm{H}) \sim 6.66 \pm 0.10 \mathrm{dex})$.
\end{abstract}

Key words. stars: abundances - stars: atmospheres - stars: early-type - galaxies: individual: Small Magellanic Cloud

\section{Introduction}

The interstellar medium (ISM) within a galaxy provides a fossil record of both stellar and galactic evolution. An excellent opportunity for investigating the metallicity dependence of such evolutionary theories is provided by the Magellanic Clouds. These dwarf irregular galaxies possess very different environments to that of the Milky Way (see Russell \& Dopita 1992), and they can be viewed in their entirety. Furthermore, the Magellanic Clouds have well established distance estimates, they are relatively unobscured by dust, and are sufficiently close to enable individual stars and nebulae to be studied in detail.

Pioneering work of SMC/LMC $\mathrm{H}_{\text {II }}$ regions by Dufour et al. (1982) established that these galaxies possess significantly lower metallicities than that found in the Sun and the Orion nebula. In recent years, the Clouds have been the subject of extensive elemental abundance investigations (see, for example, Garnett 1999; Westerlund 1997; Rolleston 1991 for a review). Abundance estimates in the Magellanic System have relied mainly upon observations of emission-line plasmas, viz. H II regions and supernova remnants (Dufour 1984; Russell \& Dopita 1990; Garnett 1999), or of supergiants (Spite et al. 1991; Hill 1999; Venn 1999a). Such objects are

Send offprint requests to: P. L. Dufton, e-mail: P.Dufton@qub.ac.uk not ideal for studying the present-day chemical composition of the ISM. For example, emission-line studies may be affected by spatial inhomogeneities in the nebula, and the fractionation of some elements from the gas phase onto dust grains; whereas, supergiant atmospheres may be contaminated by the products of nucleosynthesis that have been dredged up from the stellar interiors.

Main-sequence B-type stars (with ages typically less than $30 \mathrm{Myr}$ ) provide an excellent method for probing the metallicity and recent evolutionary history within the Milky Way and the Magellanic Clouds. Their photospheres are not normally contaminated by core processed material (see Gies \& Lambert 1992; although, see recent analyses and discussion of rotational mixing on the main-sequence by Venn et al. 2002) and their photospheric spectra are relatively rich in absorption features due to $\mathrm{C}, \mathrm{N}, \mathrm{O}, \mathrm{Mg}, \mathrm{Si}, \mathrm{S} \& \mathrm{Fe}$. Additionally, "classical" model atmosphere calculations (viz. those assuming plane parallel geometry; hydrostatic and radiative equilibrium) have been very successful in interpreting their observed spectra (see Hardorp \& Scholz 1970; Gies \& Lambert 1992; Cunha \& Lambert 1994; Kilian et al. 1994). Hence, these objects should provide unambiguous information on the present-day chemical composition of the ISM within the parent galaxy.

The motivation for this current work was the lack of nitrogen abundance estimates for unevolved stars within the SMC. Previous analyses of SMC B-type dwarfs yielded reliable 
abundance estimates for $\mathrm{C}, \mathrm{O}, \mathrm{Mg}, \mathrm{Al} \& \mathrm{Si}$ (see Dufton et al. 1990b; Rolleston et al. 1993). However, the non-detection of nitrogen absorption features in their spectra was very striking. Indeed, the model-atmosphere analyses imposed an upper constraint of $\sim 6.9$ dex on the present-day nitrogen composition of the SMC ISM - corresponding to a minimum deficiency of 0.9 dex. This is compatible with the low nitrogen abundance of $\sim 6.5$ dex observed in SMC H II regions (see Garnett 1999; Dufour 1984). To date, spectroscopic investigations of bright giants/supergiants in the SMC (see Hill 1999; Venn 1999a; Korn et al. 2000; Lennon et al. 2003) have been unable to reproduce the low nitrogen abundance observed in $\mathrm{H}$ II regions. This is not altogether surprising as the more evolved stars may be expected to have products of the $\mathrm{CN}$-cycle mixed into their stellar photospheres. Hence, high signal-to-noise ratio observations of bona-fide main-sequence stars are imperative in order to confirm the baseline abundance of nitrogen within the SMC. The field star AV 304 is an excellent target - as its main-sequence status has been previously confirmed by Dufton et al. (1990b) and Rolleston et al. (1993). Furthermore, it is exceptionally sharp-lined and hence well-suited to a detailed analysis.

It should be noted that Korn et al. (2002) performed a similar analysis of 4 B-type dwarfs in the LMC cluster NGC 2004. This work confirmed the low absolute nitrogen abundance obtained from $\mathrm{H}$ II regions to within the errors.

\section{Observations and data reductions}

High-resolution spectroscopy of AV 304 was obtained using the UV and Visual Échelle Spectrograph (D'Odorico et al. 2000) at the ESO-VLT UT2 (Kueyen) telescope on the 17th and 18th January 2001. UVES was operated with the CD\#2 cross-disperser, CCD-44 and the standard setting Blue $437 \mathrm{~nm}$, giving complete spectral coverage from approximately $\lambda \lambda 3770-4980 \AA$. The slit width was set to $1.0^{\prime \prime}$ corresponding to a resolving power of $R \approx 40000$. Given the straight-forward nature of these observations, we have made use of the pipe-line reductions. Combination of the $1 \times 2700 \mathrm{~s}$ and $1 \times 3600 \mathrm{~s}$ integrations yielded a signal-to-noise ratio of $S / N \sim 85$ in the stellar continuum.

Further manipulation of the stellar spectra, viz. radial velocity corrections, rectification of the stellar continuum and the measurement of equivalent widths for the metal and nondiffuse helium line-spectra, utilized the suite of routines provided by the spectral reduction package DIPso (Howarth et al. 1994). Full details of these methods can be found in Rolleston et al. (1999a) and references therein. The extensive equivalent width dataset for the non-diffuse helium and metal lines can be obtained from the authors upon request.

We have undertaken a comparison of the equivalent width datasets obtained here for AV 304 using the VLT/UVES+CCD with that obtained previously using the AAT/UCLES+IPCS. The comparison utilized approximately 40 measurements for unblended helium and metal lines; this is shown graphically as a $\log -\log$ plot in Fig. 1 for clarity. A least squares straight-line fit to the data points yielded a gradient of $m=0.98 \pm 0.01$; hence, there does not appear to be any significant differences

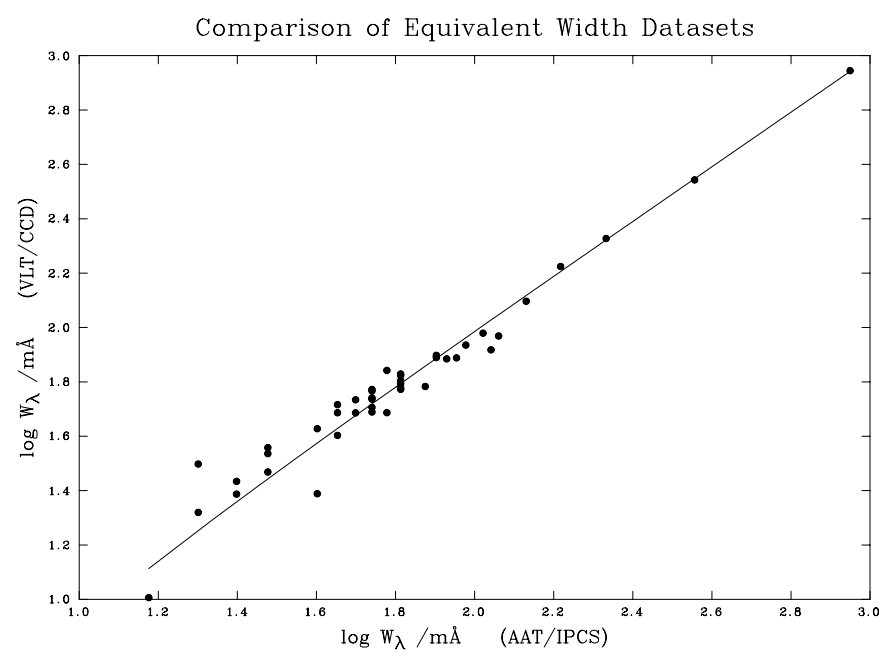

Fig. 1. Comparison of the equivalent width datasets obtained for AV 304 using the VLT/UVES and AAT/UCLES spectrographs. The solid line represents the least-squares straight-line fit to the datapoints. For clarity, the data has been presented on a log-log graph.

between the two sets of equivalent width estimates (especially for stronger lines).

\subsection{Comparison star}

High signal-to-noise échelle spectroscopy of the bright, normal Population I, B-type star HR 2387 was obtained previously with the $0.9-\mathrm{m}$ coudé feed telescope at Kitt Peak National Observatory. These data provide a spectral coverage of $\lambda \lambda 3900-4700 \AA$ at a $F W H M$ resolution of $0.1 \AA$. Stellar spectra were again extracted using similar techniques, and equivalent width estimates for the non-diffuse helium and metal-lines have been published by Hambly et al. (1997).

\section{Data analysis}

The methods used to derive the stellar atmospheric parameters are similar to those discussed by Rolleston et al. (1999a, 2000). We have adopted theoretical results based on the ATLAS9 grid of line-blanketed model-atmospheres (Kurucz 1991) together with LTE radiative transfer codes. The model-atmospheres are characterized by four parameters, viz. effective temperature $\left(T_{\text {eff }}\right)$, surface gravity $(\log g)$, microturbulence $(\xi)$ and metallicity. Determination of these parameters is an iterative process - with all steps being inter-related and $T_{\text {eff }}, \log g \& \xi$ being dependent on metallicity which is not known a priori. It should be noted that the number of iterations were generally small $(<3)$, and that the adopted atmospheric parameters (see Table 1) were deduced using models with an appropriate chemical composition.

\section{1. $T_{\text {eff }}, \log g$}

Stellar effective temperatures were derived by balancing the $\mathrm{Si}$ III/Si Iv ionization equilibrium, using absorption lines of Si III (Multiplet No. 2) 4552, 4567, $4574 \AA$ and Si IV (Multiplet No. 1) 4088, $4116 \AA$ A. Surface gravities were deduced 
Table 1. Stellar parameters.

\begin{tabular}{lll}
\hline \hline Parameter & AV 304 & HR 2387 \\
\hline$T_{\text {eff }}(\mathrm{K})$ & 27500 & 26500 \\
$\log g(\mathrm{dex})$ & 3.8 & 3.85 \\
$\xi\left(\mathrm{km} \mathrm{s}^{-1}\right)$ & 5 & 10 \\
$v \sin i\left(\mathrm{~km} \mathrm{~s}^{-1}\right)$ & 11 & 20 \\
$v_{\mathrm{lsr}}\left(\mathrm{km} \mathrm{s}^{-1}\right)$ & +129 & +15 \\
\hline
\end{tabular}

by fitting theoretical profiles to the normalized spectra of the $\mathrm{H} \epsilon, \mathrm{H} \delta, \mathrm{H} \gamma$ and $\mathrm{H} \beta$ lines. Additionally, the UVES spectra contained higher members of the Balmer series including $\mathrm{H}_{11}$, $\mathrm{H}_{10}, \mathrm{H}_{9}$ and $\mathrm{H}_{8}$. Estimates of the logarithmic surface gravity deduced from these latter features were in excellent agreement with that obtained from the lower series members. All theoretical calculations have adopted the line-broadening theory of Vidal et al. (1973).

\section{2. $\xi$}

In early B-type stars, estimates of the microturbulence parameter can be deduced from their $\mathrm{O}$ II line-spectra by removing the dependence of the derived oxygen abundance upon the observed line-strengths (see Lennon et al. 1988; Gies \& Lambert 1992). The O II line-spectra were well observed in both AV 304 and HR 2387, with reliable equivalent width estimates being measured for $\sim 100$ and $\sim 75$ transitions in each star respectively. Hence, this technique should yield robust estimates of the microturbulent velocity. A value of $5 \mathrm{~km} \mathrm{~s}^{-1}$ was obtained for AV 304 (see Fig. 2), in good agreement with that found previously from similar LTE analyses of B-type mainsequence stars (Dufton et al. 1990a; Gies \& Lambert 1992). For HR 2387, a microturbulence of $10 \mathrm{~km} \mathrm{~s}^{-1}$ was determined which is consistent with that found in a previous analysis by Hambly et al. (1997).

\subsection{Stellar $v \sin i$}

Stellar projected rotational velocities $(v \sin i)$ were estimated by convolving theoretically generated spectra with rotational broadening functions until they matched the observations. It should be noted that this procedure accounts for the effect of instrumental broadening. Our estimates are presented in Table 1.

\subsection{Photospheric abundances}

The adopted atmospheric parameters (listed in Table 1) were used to derive absolute LTE abundances for both AV 304 and HR 2387 using procedures similar to those described in Rolleston et al. (1999a, 1999b). Abundance estimates are presented in Table 3 where we have adopted a logarithmic scale with the abundance of hydrogen equivalent to 12.0 dex. Errors (where quoted) refer to the $1 \sigma$ sample standard deviations. Observational uncertainties in the equivalent width estimates were typically $\pm 5 \mathrm{~m} \AA$ - corresponding to an abundance error of $\pm 0.10 \mathrm{dex}$. Additionally, the effect of changing the atmospheric parameters by their error estimates was considered,

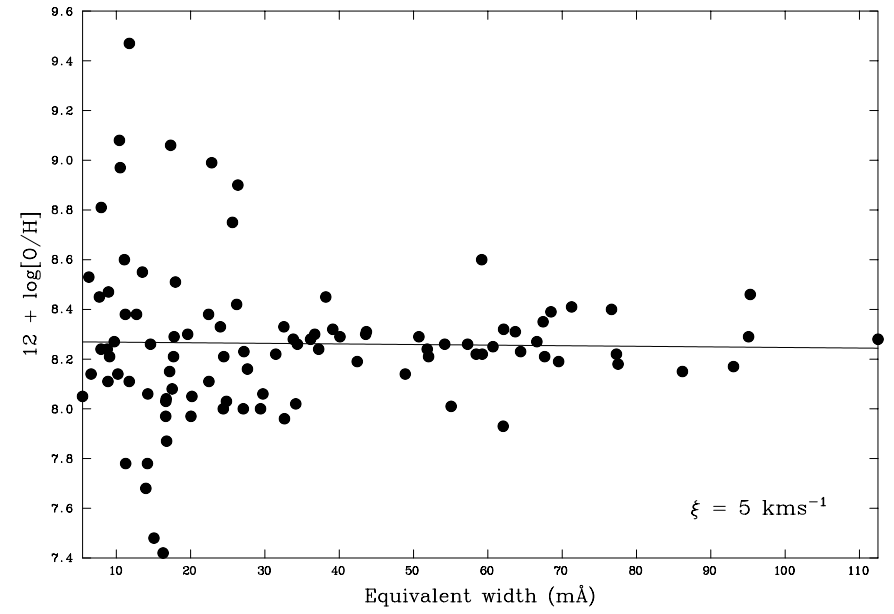

Fig. 2. AV 304 oxygen abundances derived using the atmospheric parameters presented in Table 1 as a function of equivalent width. No significant dependence is observed - indicating that the choice of $\xi=$ $5 \mathrm{~km} \mathrm{~s}^{-1}$ is appropriate.

viz. $\Delta T_{\text {eff }} \sim \pm 1000 \mathrm{~K}, \Delta \log g \sim \pm 0.1$ dex and $\triangle \xi \sim \pm 2 \mathrm{~km} \mathrm{~s}^{-1}$, and led to changes in the absolute abundance estimates by less than 0.15 dex. A detailed line-by-line differential abundance analysis was performed relative to HR 2387 for a common subset of lines with reliable equivalent width estimates. The differential abundances are presented in Tables 3 and 4. It should be noted that the effect of systematic errors in the atmospheric parameters, simplifications in the model-atmosphere analyses (e.g. the assumption of LTE), and uncertainties in the adopted atomic data will become less important for these differential results.

\subsection{SMC membership}

It is important to establish that AV 304 is indeed associated with the SMC galaxy. We used two criteria to confirm its membership, viz. stellar radial velocity, and interstellar gas absorption lines. A radial velocity estimate relative to the local standard of rest in the solar neighbourhood of $v_{\mathrm{lsr}}=+129 \mathrm{~km}^{-1}$ was deduced from the UVES spectra. This is consistent with the value of $136 \mathrm{~km} \mathrm{~s}^{-1}$ reported by Hardy et al. (1989) for the LSR velocity of the SMC. Additionally, it was possible to identify interstellar Ca II $\mathrm{H} \& \mathrm{~K}$ absorption components due to Galactic (both low and intermediate-velocity material), and SMC gas (see Fig. 3). Details of the interstellar gas components are presented in Table 2. Components E-L are almost certainly associated with the SMC; indeed, studies of SMC supergiants have identified multiple components of interstellar gas with LSR velocities between approximately $90 \leq v_{\text {lsr }} \leq$ $210 \mathrm{~km} \mathrm{~s}^{-1}$ (see Wayte 1990; Welty et al. 1997). Hence, we believe AV 304 to be a member of the SMC field.

Unfortunately, our absolute distance estimate may be subject to systematic errors - and may underestimate the true value by upto 30\% (see Rolleston et al. 1996). This is the consequence of an inadequate treatment of the Balmer lines' wings in LTE which leads to an overestimate of the stellar surface gravity. Hence, we have not included the stellar distance as a 


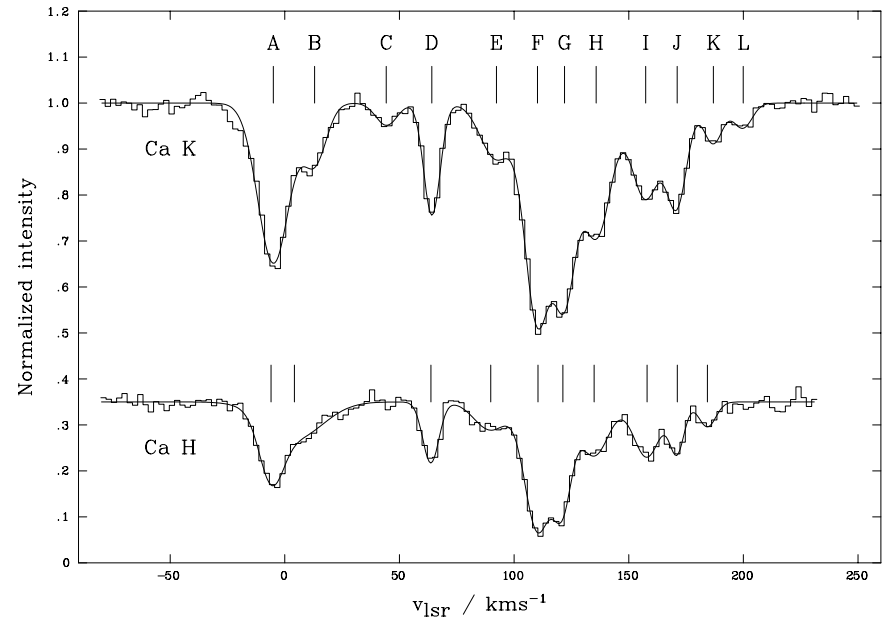

Fig. 3. Ca H\&K gas absorption towards AV 304 (histograms). Gaussian fits to the multiple components are also shown (smooth curves).

Table 2. Interstellar gas components.

\begin{tabular}{|c|c|c|c|c|}
\hline \multirow[t]{2}{*}{ Component } & \multicolumn{2}{|c|}{$\overline{\mathrm{Ca} \mathrm{K}}$} & \multicolumn{2}{|c|}{$\overline{\mathrm{CaH}}$} \\
\hline & $\begin{array}{r}v_{\text {lsr }} \\
\left(\mathrm{km} \mathrm{s}^{-1}\right)\end{array}$ & $\begin{array}{r}W_{\lambda} \\
(\mathrm{m} \AA)\end{array}$ & $\begin{array}{r}v_{\mathrm{lsr}} \\
\left(\mathrm{km} \mathrm{s}^{-1}\right)\end{array}$ & $\begin{array}{r}W_{\lambda} \\
(\mathrm{m} \AA)\end{array}$ \\
\hline \multicolumn{5}{|l|}{ Galactic LVC } \\
\hline A.... & -5.0 & 78 & -6.0 & 21 \\
\hline В.... & +13.0 & 22 & +4.2 & 33 \\
\hline \multicolumn{5}{|l|}{ Galactic IVC } \\
\hline $\mathrm{C} \ldots .$. & +44.2 & 7 & - & - \\
\hline D.... & +64.1 & 29 & +63.7 & 15 \\
\hline \multicolumn{5}{|l|}{ SMC } \\
\hline E.... & +92.3 & 28 & +89.8 & 14 \\
\hline F.... & +110.2 & 84 & +110.4 & 52 \\
\hline G.... & +122.0 & 60 & +121.3 & 27 \\
\hline Н.... & +135.8 & 61 & +134.9 & 25 \\
\hline $\mathrm{I} \ldots .$. & +157.4 & 41 & +158.0 & 23 \\
\hline $\mathrm{J} \ldots .$. & +171.1 & 31 & +171.2 & 11 \\
\hline $\mathrm{K} \ldots .$. & +186.9 & 12 & +184.3 & 7 \\
\hline L.... & +199.9 & 7 & - & - \\
\hline
\end{tabular}

primary membership criterion, although this is clearly compatible with AV 304 being associated with the SMC.

\section{Photospheric composition of AV 304}

As a consequence of the very low projected rotational velocity $\left(\sim 11 \mathrm{~km} \mathrm{~s}^{-1}\right)$ observed for AV 304 together with the earlyB spectral type, approximately 176 absorption features were identified in the $\lambda \lambda 3770-4980 \AA$ region. Furthermore, it was possible to reliably model $\sim 150$ of these transitions in the absolute abundance analysis, and $\sim 100$ lines were used to derive differential abundances relative to HR 2387. Example regions of the UVES spectra are shown in Fig. 4. Specific comments relevant to individual elements are discussed below.
Table 3. Absolute and differential abundance analysis of AV 304.

\begin{tabular}{lcrcr}
\hline \hline Species & \multicolumn{1}{c}{$12+\log (\mathrm{X} / \mathrm{H})$} & \multicolumn{1}{c}{$\Delta[\mathrm{X} / \mathrm{H}]$} \\
\hline $\mathrm{He}_{\text {I }}$ & $10.86 \pm 0.05$ & $(4)$ & $-0.01 \pm 0.04$ & $(3)$ \\
$\mathrm{C}_{\text {II }}$ & $7.00 \pm 0.18$ & $(3)$ & $-0.58 \pm 0.11$ & $(2)$ \\
$\mathrm{C}_{\text {III }}$ & $7.41 \pm 0.23$ & $(6)$ & $-0.78 \pm 0.14$ & $(4)$ \\
$\mathrm{N}_{\text {II }}$ & $6.66 \pm 0.01$ & $(2)$ & $-1.17 \pm 0.08$ & $(2)$ \\
$\mathrm{O}_{\text {II }}$ & $8.23 \pm 0.33$ & $(99)$ & $-0.38 \pm 0.09$ & $(68)$ \\
$\mathrm{Ne}_{\text {II }}$ & $7.80 \pm 0.07$ & $(2)$ & $-0.42 \pm 0.02$ & $(2)$ \\
$\mathrm{Mg}_{\text {II }}$ & 6.79 & $(1)$ & -0.52 & $(1)$ \\
$\mathrm{Al}_{\text {III }}$ & $5.34 \pm 0.15$ & $(2)$ & $-0.72 \pm 0.09$ & $(2)$ \\
$\mathrm{Si}_{\text {III }}$ & $6.79 \pm 0.05$ & $(3)$ & $-0.50 \pm 0.03$ & $(2)$ \\
$\mathrm{Si}_{\text {IV }}$ & $6.79 \pm 0.04$ & $(2)$ & -0.46 & $(1)$ \\
$\mathrm{S}_{\text {III }}$ & $6.44 \pm 0.50$ & $(8)$ & $-0.42 \pm 0.09$ & $(6)$ \\
$\mathrm{Fe}_{\text {III }}$ & $6.62 \pm 0.26$ & $(3)$ & $-0.50 \pm 0.19$ & $(3)$ \\
\hline
\end{tabular}

1. Errors (where quoted) refer to the $1 \sigma$ standard deviation of abundance estimates obtained from individual lines. Uncertainties on the single measurements should be typically less than 0.2 dex (see Sect. 3.4).

2. The numbers in parentheses after each abundance estimate represent the number of absorption lines used in the model-atmosphere analysis.

3. $\Delta[\mathrm{X} / \mathrm{H}]=\log (\mathrm{X} / \mathrm{H})_{\mathrm{AV}} 304-\log (\mathrm{X} / \mathrm{H})_{\mathrm{HR}} 2387$.

\subsection{Helium}

The neutral helium line-spectrum was well observed in AV 304 - permitting absolute abundance estimates to be derived for 8 diffuse and 4 non-diffuse lines. A direct comparison of the line profiles for AV 304 and the Galactic standard demonstrated their similar helium line-spectra and, hence, photospheric compositions. Indeed, the model-atmosphere analysis yielded a differential helium abundance of $-0.01 \pm$ 0.04 dex. This provides indirect evidence that the photosphere of AV 304 has not been contaminated by products of nucleosynthesis mixed from the interior, and hence should be a reliable tracer of the chemical composition of the natal environment.

\subsection{Carbon}

Estimates of the photospheric carbon composition were determined using lines from two ionization stages, viz. $\mathrm{C}_{\text {II }} / \mathrm{C}_{\text {III }}$. Mean absolute values obtained from each ion appear to be discrepant by $\sim 0.4$ dex (see Table 3 ); however, this difference and the relatively large scatter in the individual abundance estimates used to derive these mean values may be partially attributable to non-LTE effects. For example, the $\mathrm{C}_{\text {II }}$ line-spectrum contains the $4267 \AA$ transition for which Eber $\&$ Butler (1988) have reported significant differences between LTE and non-LTE line-formation calculations. Additionally, differences were found between the absolute abundance estimates obtained using lines from different atomic multiplets, viz. C III (Multiplet No. 1) 4647, 4650, $4651 \AA$ and $\mathrm{C}_{\text {III }}$ (Multiplet No. 16) 4069, $4070 \AA$.

By contrast, the results of the differential analysis (see Table 3) re-inforce the comments made in Sect. 3.4, that nonLTE effects and the choice of atomic data will become less 

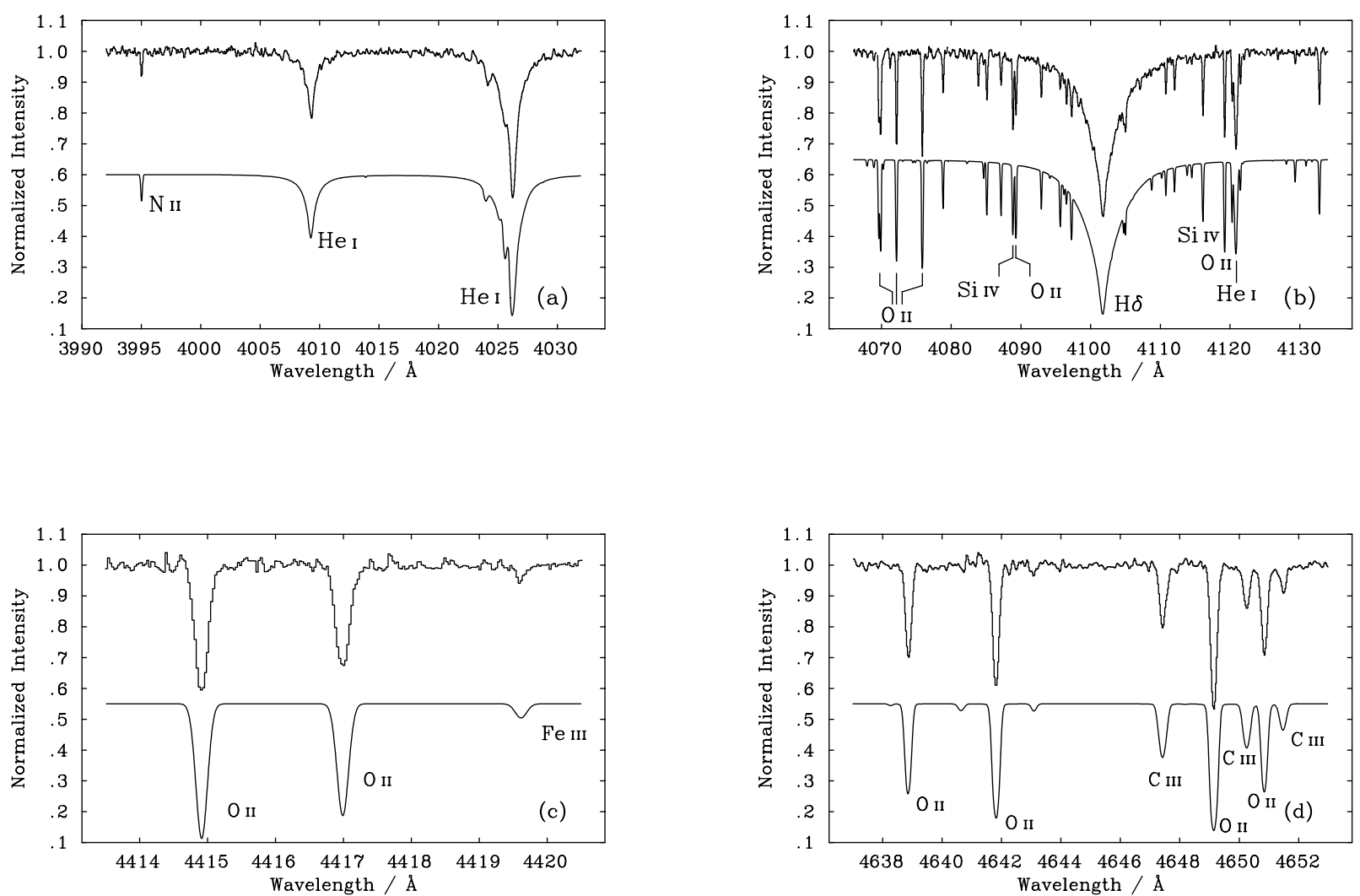

Fig. 4. Examples of the observed spectra (histograms) obtained for AV 304 using UVES on the VLT-UT2, for spectral regions including a) neutral helium features, b) $\mathrm{H} \delta$ profile, and c), d) numerous metal lines. The synthesized spectra (smooth curves) have been generated using the adopted atmospheric parameters derived for AV 304 (see Table 1) and the absolute abundances presented in Table 3. Excellent agreement is found between observation and theory.

significant in such a comparison. Indeed, the errors associated with the mean abundance estimates deduced from $\mathrm{C}_{\text {II }}$ and $\mathrm{C}_{\text {III }}$ lines, and the difference between these mean values was reduced significantly (by a factor of 2). Thus, these results are consistent with AV 304 having a mean carbon underabundance of -0.65 dex.

\subsection{Nitrogen}

The significance of determining a nitrogen abundance in the atmosphere of an unevolved, SMC main-sequence star can not be overstated. Equivalent width estimates were obtained for $2 \mathrm{~N}_{\text {II }}$ lines in AV 304 which yielded an absolute nitrogen abundance of $6.66 \pm 0.01 \mathrm{dex}$. This result should be reliable as non-LTE effects have been found to be relatively small $(<0.1$ dex) for the N II species (see Becker \& Butler 1989). Furthermore, the intrinsic weakness of the $\mathrm{N}_{\text {II }}$ line-spectrum reduced the effect of errors in the adopted atmospheric parameters on the resultant nitrogen abundance determinations. Hence, we believe that our nitrogen abundance estimate is accurate to \pm 0.1 dex. Using similar methods to those described in Rolleston et al. (1993) and Mooney et al. (2001), we obtained upper limits on the equivalent widths of a further $5 \mathrm{~N}$ II features. These constraints are also compatible with an absolute nitrogen abundance of 6.66 dex. The differential analysis inferred a nitrogen deficiency of $-1.17 \pm 0.08$ dex. This confirms and quantifies the conclusions of Dufton et al. (1990b) and Rolleston et al. (1993) that AV 304 appeared to exhibit a greater nitrogen underabundance relative to the other metals (see Sect. 5).

\subsection{Oxygen, silicon, sulphur}

Approximately $80 \%$ of the absorption features observed in the spectra of AV 304 arise from transitions of the $\alpha$-elements oxygen, silicon and sulphur. This is dominated by the plethora of $\sim 100$ O II lines. Although non-LTE effects have been observed to be small for this species (see Becker \& Butler 1988), the standard deviation associated with the mean oxygen abundance is relatively large (viz. $0.3 \mathrm{dex}$ ). This is unlikely to be due to observational uncertainties in the equivalent width estimates given the quality of the UVES spectra. Rather, it may reflect errors in the adopted atomic data, particularly for those weaker lines (see Fig. 2) which are less well studied. Indeed, the results of the differential analysis (where the choice of the atomic data is less important) supports this. Using 68 absorption lines, we find a mean differential oxygen abundance of -0.38 dex with a scatter of 0.09 dex.

Equivalent widths were measured for $8 \mathrm{Si}$ III and $4 \mathrm{Si}$ IV lines in the UVES spectra of AV 304. However, only the strongest lines were observed in the standard HR 2387 (viz. Si III 4552, 4567, $4574 \AA$ \& \& Si Iv 4088, $4116 \AA$ ). In order to 
Table 4. Differential abundances relative to iron.

\begin{tabular}{llc}
\hline \hline Element & $\Delta[\mathrm{X} / \mathrm{Fe}]$ \\
\cline { 2 - 3 } $\mathrm{C}$ & -0.18 \\
$\mathrm{~N}$ & -0.67 \\
$\mathrm{O}$ & +0.12 \\
$\mathrm{Ne}$ & +0.08 \\
$\mathrm{Mg}$ & -0.02 \\
$\mathrm{Al}$ & -0.22 \\
$\mathrm{Si}$ & +0.02 \\
$\mathrm{~S}$ & +0.08 \\
\hline
\end{tabular}

1. $\Delta[\mathrm{X} / \mathrm{Fe}]=\log (\mathrm{X} / \mathrm{Fe})_{\mathrm{AV} 304}-\log (\mathrm{X} / \mathrm{Fe})_{\mathrm{HR} 2387}$.

maintain consistency between the model-atmosphere analyses of both stars, the above subset of lines was used in the determination of the stellar effective temperatures (see Sect. 3.1). Abundance estimates presented in Table 3 were derived using these transitions. However, it should be noted that including the full silicon dataset in the abundance analysis does not significantly alter the results; indeed, abundance estimates deduced for the Si III and Si Iv lines change by $\sim 0.1$ and 0.05 dex respectively.

Determination of a sulphur abundance was based on $8 \mathrm{~S} \mathrm{III}$ lines. Again, a relatively large scatter is observed in the estimates obtained from individual features. By contrast, the differential treatment yielded a relatively well constrained mean sulphur deficiency of $-0.42 \pm 0.09 \mathrm{dex}-$ suggesting that the atomic data may be a source of the larger absolute abundance scatter.

\subsection{Neon, magnesium, aluminium}

Absolute abundance estimates for neon, magnesium and aluminium were based on $2 \mathrm{Ne}$ II, $1 \mathrm{Mg}$ II and $2 \mathrm{Al}$ III absorption lines respectively. The neon and aluminium features are weak with line-strengths of $\sim 10 \mathrm{~m} \AA$, while the $\mathrm{Mg}_{\text {II }} 4481 \AA$ doublet is well observed, $W_{\lambda} \sim 49 \mathrm{m \AA}$. Neon and magnesium display underabundances of -0.42 and -0.52 dex respectively. Results for these elements, and in particular the greater underabundance of $-0.72 \pm 0.09$ dex observed for aluminium, should be treated with caution.

\subsection{Iron}

The optical spectrum of iron in early B-type dwarfs is intrinsically weak (see Gies \& Lambert 1992; Rolleston et al. 2000) for reasons of its atomic structure. Moreover, the subsolar metallicity of the SMC has to date precluded the observation of iron absorption features in the spectra of such stars (see Rolleston et al. 1993; Korn et al. 2000). Given that iron has a different site of nucleosynthetic origin than the $\alpha$-elements, the determination of abundance estimates for both iron and the $\alpha$-elements in the photosphere of a SMC dwarf is significant.

Three Fe III absorption lines (with equivalent widths between 7-10 $\mathrm{m \AA}$ ) were observed in the UVES spectra of AV 304. These yield a differential iron abundance of $-0.50 \pm$ 0.19 dex.

\section{Present-day SMC abundance pattern}

The results of the differential abundance analysis can be summarized as follows. AV 304 displays a normal helium composition and a mean metallicity of $-0.44 \pm 0.06$ dex as deduced using the $\alpha$-elements $(\mathrm{O}, \mathrm{Ne}, \mathrm{Mg}, \mathrm{Si} \& \mathrm{~S})$. The latter result does not change if the 3 best observed elements are only considered, viz. $-0.43 \pm 0.05$ dex for $\mathrm{O}, \mathrm{Si} \& \mathrm{~S}$. Relative to iron $(\triangle[\mathrm{Fe} / \mathrm{H}] \sim$ -0.50 dex), oxygen may be slightly overabundant, whereas carbon and aluminium may be underabundant by $\sim-0.2$ dex. The most striking result presented in Tables 3 and 4 is the large underabundance of nitrogen relative to both hydrogen (of approximately $-1.2 \mathrm{dex}$ ) and to iron (of approximately $-0.7 \mathrm{dex}$ ). We note that Dufton et al. (1990b) were unable to determine an abundance for this element in their analysis of the spectra of AV 304 due to the intrinsic weakness of the $\mathrm{N}$ ir line-spectra.

Given the quality of the UVES dataset, a detailed comparison of the elemental abundances derived for different types of SMC objects is merited. A diverse range of objects have been used to determine the present-day chemical composition of the SMC including H II regions, B-type dwarfs/giants, B-, A-, F-, G- \& K-type supergiants and Cepheids. Abundance analyses have utilized a range of modelling techniques/codes and atomic/molecular transitions, different spectral regions and spectral resolutions and different sources of atomic data. These may lead to systematic differences between the sets of results. For example, whereas it may be appropriate to compare results derived for late-type supergiants with solar values, B-type dwarfs possess effective temperatures $20000 \mathrm{~K}$ hotter. Moreover, their spectra are dominated by lines of single and double ionized metals, whereas cool stars display absorption features due to neutral metals and molecular compounds. Indeed, it has been recognized by others that such problems can be circumvented if SMC objects are analysed using identical methods relative to "like" Galactic objects (see Pagel 1992; Venn 1999a). We have produced a compilation of differential abundance estimates for SMC objects, where $\Delta[\mathrm{X} / \mathrm{H}]=$ $\log (\mathrm{X} / \mathrm{H})_{\text {OBJECT }}-\log (\mathrm{X} / \mathrm{H})_{\text {GALACTIC. These results are pre- }}$ sented in Table 5, where details of the individual investigations and the Galactic analogues are provided in the footnotes.

\subsection{Comparison with stellar studies}

The chemical composition of AV 304 can be compared with that found previously for the SMC. The only other studies of near main sequence B-type stars has been the previous analysis of AV 304 by Dufton et al. (1990b) and of four targets by Rolleston et al. (1993). Additionally there are several studies (see, for example, Korn et al. 2000; Dufton et al. 2000; Lennon et al. 2003) of B-type giants and supergiants. However, the atmospheres of even the giants appear to be contaminated with the products of nucleosynthetic processing (Korn et al. 2000; Lennon et al. 2003); hence, they are not suitable for estimating the composition of CNO in the SMC interstellar medium. Furthermore, there have been numerous analyses 
Table 5. Comparison of SMC objects relative to "like" Galactic objects.

\begin{tabular}{|c|c|c|c|c|c|c|c|}
\hline \multirow[t]{3}{*}{ Element } & \multirow{3}{*}{$\frac{\text { Dwarfs }}{\text { AV } 304}$} & \multirow{3}{*}{$\frac{\text { Giants }}{\text { B IV }}$} & \multicolumn{4}{|l|}{ 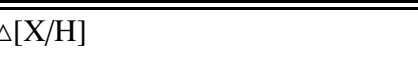 } & \multirow{3}{*}{$\frac{\text { Nebulae }}{\mathrm{H}_{\text {II }}}$} \\
\hline & & & \multicolumn{4}{|c|}{ Supergiants } & \\
\hline & & & $\mathrm{B}$ I/II & $\mathrm{A}_{\mathrm{I}}$ & $\mathrm{FG}_{\mathrm{I}}$ & $\mathrm{K}_{\mathrm{I}}$ & \\
\hline $\mathrm{C}$ & -0.68 & -0.61 & -0.85 & - & -0.60 & -0.37 & - \\
\hline $\mathrm{N}$ & -1.17 & +0.17 & -0.55 & -0.72 & - & -1.62 & -1.03 \\
\hline $\mathrm{O}$ & -0.38 & -0.59 & -0.48 & -0.45 & -0.74 & -0.69 & -0.64 \\
\hline $\mathrm{Ne}$ & -0.42 & - & - & - & - & - & -0.57 \\
\hline $\mathrm{Mg}$ & -0.52 & -0.48 & -0.76 & -0.65 & -0.73 & - & - \\
\hline $\mathrm{Al}$ & -0.72 & - & -0.72 & - & -0.69 & -0.65 & - \\
\hline $\mathrm{Si}$ & -0.48 & -0.57 & -0.53 & -0.36 & -0.79 & - & - \\
\hline$S$ & -0.42 & - & - & - & -0.94 & - & -0.67 \\
\hline $\mathrm{Fe}$ & -0.50 & - & -0.43 & -0.78 & -0.70 & -0.59 & - \\
\hline
\end{tabular}

Differential abundances were obtained or derived from the following data sources.

AV 304: This paper.

B Iv: $\quad$ Lennon et al. (1996, 2003).

B I/II: Dufton et al. (2000); Korn et al. (2000) - compared to the analyses of Galactic B-type dwarfs by the same German group, viz. Gummersbach et al. (1998) \& Kaufer et al. (1994). It should be noted that a transition to non-LTE analyses was made for the study by Korn et al.; hence, it is not a true differential analysis. However, such a comparison should not affect the numbers significantly - Korn (private communication.)

A I: $\quad$ Venn (1999a).

FG I: $\quad$ Russell \& Bessell (1989); Spite et al. (1989, 1991); Spite \& Spite (1990); Barbuy et al. (1991); Luck et al. (1998).

K I: $\quad$ Gonzalez \& Wallerstein (1999) - compared to a study of hot M-supergiants in the Galactic cluster h \& $\chi$ Persei (Gonzalez \& Wallerstein 2000).

H II regions: $\quad$ Studies by Dufour (1984), Russell \& Dopita (1992), Reyes (1999), Kurt et al. (1999) and Testor (2001) were compared with Galactic values in Orion and the Solar neighbourhood (Dufour et al. 1982; Shaver et al. 1983). Peimbert et al. (2000) analysed their SMC H II region sample relative to M 17 (Peimbert et al. 1992; Esteban et al. 1999).

Table 6. Comparison of absolute abundances in the SMC.

\begin{tabular}{|c|c|c|c|c|c|c|c|}
\hline \multirow[t]{3}{*}{ Elem } & \multirow{3}{*}{$\begin{array}{l}\text { Dwarfs } \\
\text { AV } 304\end{array}$} & \multicolumn{5}{|c|}{$\epsilon(\mathrm{X})$} & \multirow{3}{*}{$\frac{\text { Nebulae }}{\mathrm{H}_{\text {II }}}$} \\
\hline & & \multirow{2}{*}{$\frac{\text { Giants }}{\text { B IV }}$} & \multicolumn{4}{|c|}{ Supergiants } & \\
\hline & & & $\mathrm{B}_{\mathrm{I} / \mathrm{II}}$ & $\mathrm{A}_{\mathrm{I}}$ & $\mathrm{FG}_{\mathrm{I}}$ & $\mathrm{K}_{\mathrm{I}}$ & \\
\hline $\mathrm{C}$ & 7.20 & 6.85 & 7.35 & - & 7.75 & 7.52 & 7.40 \\
\hline $\mathrm{N}$ & 6.66 & 7.62 & 7.17 & 7.33 & & 7.14 & 6.56 \\
\hline $\mathrm{O}$ & 8.23 & 8.05 & 8.05 & 8.40 & 8.01 & 7.88 & 8.05 \\
\hline $\mathrm{Ne}$ & 7.80 & - & - & - & - & - & 7.31 \\
\hline $\mathrm{Mg}$ & 6.79 & 6.65 & 6.78 & 6.82 & 6.88 & 6.82 & - \\
\hline $\mathrm{Al}$ & 5.34 & - & 5.58 & - & - & 5.55 & - \\
\hline $\mathrm{Si}$ & 6.79 & 6.63 & 6.81 & 6.97 & 7.00 & 6.75 & - \\
\hline S & 6.44 & - & - & - & 6.45 & - & 6.46 \\
\hline $\mathrm{Fe}$ & 6.20 & - & 6.82 & 6.70 & 6.78 & 6.65 & - \\
\hline
\end{tabular}

Abundances estimates have been obtained from the following data sources.
AV 304:
This paper.
B Iv: $\quad$ Lennon et al. (1996, 2003).
B I/II: $\quad$ Dufton et al. (2000); Korn et al. (2000).
A I: $\quad$ Venn (1999a).
FG I: $\quad$ Russell \& Bessell (1989); Spite et al. (1989, 1991); Spite \& Spite (1990); Barbuy et al. (1991).
K I: $\quad$ Barbuy et al. (1991); Spite et al. (1991); Hill (1997); Hill et al. (1997); Hill (1999); Gonzalez \& Wallerstein (1999).
H II regions: $\quad$ Russell \& Dopita (1992); Reyes (1999); Garnett (1999); Kurt et al. (1999); Peimbert et al. (2000); Testor (2001). 
of evolved, late-type supergiants to estimate chemical compositions in the SMC (see Table 5 and references therein). Below we discuss key elements in more detail.

\subsubsection{Iron}

AV 304 displays an iron underabundance which is in excellent agreement with that found for 3 B-type bright giants (see Korn et al. 2000). Indeed, the scatter between individual SMC analyses is relatively small $( \pm 0.17 \mathrm{dex})$. However, these results for the B-stars are at variance with the large deficiency observed in AFG-supergiants. This is not expected given that the B-types evolve into the latter. However, it must be noted that the iron abundances in B-types were based on weak (and relatively few) absorption lines.

\subsubsection{Carbon}

The photospheric carbon abundance of B-type dwarfs should not have been altered by the mixing of $\mathrm{CN}$-cycled gas; the same may not be true of the more evolved, Galactic/SMC supergiants. There has been some discussion in the literature (see Venn 1999a) about discrepancies in the absolute carbon abundances deduced for $\mathrm{F}-\mathrm{K}$ supergiants $(\sim 7.7$ dex $)$ and nebular/B-type stars $(\sim 7.3 \mathrm{dex})$. Indeed, this higher carbon abundance estimate obtained for the former is evident in our compilation of absolute SMC abundances (see Table 6). However, it is worthwhile noting that the comparison of SMC objects relative to "like" Galactic objects does not display any significant differences for this element. Indeed, the deficiency of -0.68 dex observed for AV 304 is in good agreement with that found for F-K supergiants (viz. $-0.60 \mathrm{dex}$ ).

\subsubsection{Nitrogen}

The large scatter observed between the nitrogen abundance estimates (see Table 5) is almost certainly indicative of the different degrees of mixing in the atmospheres of the various groups of stars. Our determination of a nitrogen abundance for AV 304 is in excellent agreement with the deficiency of -1.03 dex found in SMC $\mathrm{H}$ II regions. Comparison with the values obtained for more evolved B-type and A-type supergiants illustrates the amount of $\mathrm{CN}$-cycled material mixed into their atmospheres (see, for example, Schönberner 1988; Venn 1999b). Given the low base-line nitrogen abundance of the SMC (see Sect. 5.2), a small amount of carbon nuclei converted into Nitrogen nuclei via the $\mathrm{CN}$-cycle results in a large fractional increase in the total number of nitrogen nuclei. For example, a 0.1 dex decrease in the photospheric carbon abundance of 7.4 dex would result in a 0.4 dex increase in the photospheric nitrogen abundance from 6.6 dex to 7.0 dex.

There is evidence that the nitrogen abundance in SMC B-type giants can be significantly enhanced due to the mixing of nucleosynthetic processed material to the stellar surface (see Lennon et al. 2003 and references therein). However, the large gravity estimated for AV 304 (coupled with the very low value for its nitrogen abundance estimate) implies that the value deduced here probably represents that of the contemporary SMC interstellar medium.

\subsection{4. $\alpha$-elements}

AV 304 appears to display a marginal overabundance $(-0.45 \mathrm{dex})$ in the $\alpha$-elements $(\mathrm{O}, \mathrm{Ne}, \mathrm{Mg}, \mathrm{Si} \& \mathrm{~S})$ relative to the mean value obtained for the SMC (viz. $-0.63 \mathrm{dex}$ ). It should be noted that these numbers do not change siginificantly if only the 3 best observed elements $(\mathrm{O}, \mathrm{Mg} \& \mathrm{Si})$ are considered. Good agreement is obtained between AV 304 and the analysis of near main-sequence stars in NGC 330 (see Lennon et al. 2003); the latter investigation yielded a mean $\alpha$-element deficiency of $-0.55 \mathrm{dex}$. Furthermore, the $\Delta[\alpha / \mathrm{Fe}]$ abundance ratio deduced for AV 304 of +0.05 dex is in excellent agreement with the value found in A-type supergiants, viz. $\Delta[\mathrm{O} / \mathrm{Fe}] \sim+0.1$ dex. Indeed, this range of values is predicted by model simulations of the chemical evolution of the SMC (see, for example, Pagel \& Tautvaišienè 1998).

\subsubsection{Aluminium}

It is worthwhile noting that excellent agreement is found for this element between the different classes of objects. The larger deficiency of aluminium relative to iron of -0.15 dex may indeed be real.

\subsection{Comparison with $H$ II regions}

For many of the objects studied, the chemical composition (and in particular, the CNO abundance pattern) will have been modified during their evolution. By contrast, the photospheric composition of main-sequence stars such as AV 304 should be directly comparable to the results for $\mathrm{H}$ II regions. Indeed, our analysis of AV 304 is the first stellar investigation to reproduce the large deficiency and low absolute abundance for nitrogen that is observed in SMC H II regions.

The determination of initial (or base-line) abundances in the SMC is imperative, if $\mathrm{CNO}$ abundances in Magellanic Cloud stars are to be interpreted correctly in the context of stellar evolution (Venn 1999a). Since these elements have different nucleosynthetic sites from each other and from Fe-group elements, it is not sufficient to assume that they scale with the overall metal underabundance of the SMC. Hence, we believe that it is worthwhile to compare our absolute abundance estimates of CNO \& S with nebular results. In Table 6 , we present a compilation of published absolute abundance estimates for different groups of objects in the SMC.

Analyses of SMC H II regions (see, for example, Dufour 1984; Russell \& Dopita 1990; Garnett et al. 1995 and Kurt et al. 1999) yield abundances of 7.4 dex for carbon, $6.6 \mathrm{dex}$ for nitrogen and 8.1 dex for oxygen. For the latter two elements, these are in excellent agreement with the absolute LTE values obtained here. This is not surprising as non-LTE effects for the $\mathrm{N}_{\text {II }}$ and $\mathrm{O}_{\text {II }}$ ions are known to be small (see Becker \& Butler 1988, 1989). For carbon, there is excellent agreement between the nebular value and that deduced from $\mathrm{C}$ III, 
but a difference of 0.4 dex with that deduced from $\mathrm{C}_{\text {II. }}$. As discussed by Lennon et al. (2003), this ion is susceptible to nonLTE effects and correcting for these effects would bring the two values into excellent agreement. Similarly, absolute abundance estimates for Sulphur yielded excellent agreement (see Table 6).

Comparison of the absolute abundances for AV 304 and the $\mathrm{H}$ II regions infer depletions from the gas phase onto dust grains of approximately $0.1,0.2$ and 0.0 dex for nitrogen, oxygen and sulphur respectively; in good agreement with that found for the low-density, low metallicity environment of the Galactic halo. Although, we are confident that our absolute abundances for nitrogen and oxygen are indicative of the baseline SMC composition for these elements, dust depletion fractions of $<0.2$ dex should be treated with caution as non-LTE effects are of the order of 0.1 dex for these species.

\subsection{Comparison of $\log (N / O)$ ratio in the Galaxy, $L M C$, $S M C$}

It is worthwhile commenting on the $\log (\mathrm{N} / \mathrm{O})$ abundance ratios obtained for the SMC, LMC and the Galaxy. Using our results for AV 304, viz. a mean Nitrogen abundance of 6.66 dex and an oxygen value of 8.23 dex, we obtain $\log (\mathrm{N} / \mathrm{O})=-1.57 \mathrm{dex}$. This compares with a $\log (\mathrm{N} / \mathrm{O})$ ratio of $-1.36 \mathrm{dex}$ (corresponding to an oxygen abundance of $8.4 \mathrm{dex}$ ) in the LMC (Korn et al. 2002) and $\log (\mathrm{N} / \mathrm{O})=-0.87 \mathrm{dex}$ (corresponding to an oxygen abundance of $8.68 \mathrm{dex}$ ) in the solar neighbourhood (Gies $\&$ Lambert 1992). A comparison of these values can be made with chemical evolution models (see, for example, Henry et al. 2000). In this latter study, the models satisfactorily reproduced the behaviour of the $\mathrm{N} / \mathrm{O}$ and $\mathrm{C} / \mathrm{O}$ abundance ratios as a function of metallicity (based on a large sample of Galactic and extragalactic $\mathrm{H}$ II region data). Whereas it would appear that secondary production dominates the evolution of nitrogen in the Galactic disk out to a Galactocentric radius of $17 \mathrm{kpc}$ (see Rolleston et al. 2000), the present-day nitrogen composition of the SMC is dominated by the primary production in intermediate mass stars. Korn et al. (2002) also concluded that this was the case for the LMC.

\section{Conclusions}

1. AV 304's membership of the SMC field was confirmed using stellar radial velocities and the observation of interstellar Ca H\&K absorption features due to SMC gas in the stellar spectra.

2. The subsequent model-atmosphere analysis of the UVES spectra confirmed that AV 304 is indeed a bona-fide mainsequence star, with a photospheric composition that should be representative of the present-day SMC ISM.

3. Analysis of AV 304 infers a present-day SMC metallicity of $-0.44 \pm 0.05$ dex as derived using the $\alpha$-elements $(\mathrm{O}, \mathrm{Mg}$ $\& \mathrm{Si})$, and an iron deficiency of -0.5 dex.

4. Relative to iron, oxygen was found to be overabundant by $\sim 0.1$ dex, whilst carbon and aluminium displayed a larger underabundance of $\sim 0.2$ dex.
5. The most striking result was the large deficiency observed for nitrogen, viz. $\Delta[\mathrm{N} / \mathrm{H}] \sim-1.2$ dex and $\Delta[\mathrm{N} / \mathrm{Fe}] \sim$ -0.7 dex. This is in excellent agreement with that observed in SMC H II regions and almost certainly represents the present-day base-line Nitrogen composition within the SMC ISM.

6. The present-day evolution of nitrogen in the SMC is dominated by primary production in intermediate mass stars.

7. Given the quality of the observational data and the reliablity of (at least) the differential abundance analysis, these abundances are among the most reliable currently available for the SMC.

Acknowledgements. We would like to thank the staffs of the European Southern Observatory and Kitt Peak National Observatory for their assistance in obtaining the observational data. Data reduction and analysis was performed on the PPARC funded N. Ireland STARLINK node. WRJR acknowledges financial assistance from the PPARC. KAV would like to thank the National Science Foundation for funding through a CAREER/PECASE award, AST-9984073. Finally, we would like to thank S. Smartt and the referee for valuable comments on the initial version.

\section{References}

Barbuy, B., Spite, M., Spite, F., \& Milone, A. 1991, A\&A, 247, 15

Becker, S. R., \& Butler, K. 1988, A\&A, 201, 232

Becker, S. R., \& Butler, K. 1989, A\&A, 209, 244

Cunha, K., \& Lambert, D. L. 1994, ApJ, 426, 170

D'Odorico, S., Cristiani, S., Dekker, H., et al. 2000, in SPIE 4005 Conf., 121

Dufour, R. J. 1984, IAU Symp., 108, 353

Dufour, R. J., Shields, G. A., \& Talbot, R. J. 1982, ApJ, 252, 461

Dufton, P. L., Brown, P. J. F., Fitzsimmons, A., \& Lennon, D. J. 1990a, A\&A, 232, 431

Dufton, P. L., Fitzsimmons, A., \& Howarth, I. D. 1990b, ApJ, 362, L59

Dufton, P. L., McErlean, N. D., Lennon, D. J., \& Ryans, R. S. I. 2000, A\&A, 293, 710

Eber, F., \& Butler, K. 1988, A\&A, 202, 153

Esteban, C., Peimbert, M., Torres-Peimbert, S., \& Garcia-Rojas, J. 1999, RMXAA, 35, 85

Garnett, D. R. 1999, IAU Symp., 190, 266

Garnett, D. R., Skillman, E. D., Dufour, R. J., et al. 1995, ApJ, 443, 64

Gies, D. R., \& Lambert, D. L. 1992, ApJ, 387, 673

Gonzalez, G., \& Wallerstein, G. 1999, AJ, 117, 2286

Gonzalez, G., \& Wallerstein, G. 2000, AJ, 119, 1839

Gummersbach, C. A., Kaufer, A., Schäfer, D. R., Szeifert, T., \& Wolf, B. 1998, A\&A, 338, 881

Hambly, N. C., Rolleston, W. R. J., Keenan, F. P., Dufton, P. L., \& Saffer, R. A. 1997, ApJS, 111, 419

Hardorp, J., \& Scholz, M. 1970, ApJ, 154, 1111

Hardy, E., Suntzeff, N. B., \& Azzopardi, M. 1989, ApJ, 344, 210

Henry, R. B. C., Edmunds, M. G., \& Köppen, J. 2000, ApJ, 541, 660

Hill, V. 1997, A\&A, 324, 435

Hill, V. 1999, A\&A, 345, 430

Hill, V., Barbuy, B., \& Spite, M. 1997, A\&A, 323, 461

Howarth, I. D., Murray, J., \& Mills, D. 1994, Starlink User Note, No. 50.15

Kaufer, A., Szeifert, T., Krenzin, R., Baschek, B., \& Wolf, B. 1994, A\&A, 289, 740 
Kilian, J., Montenbruch, O., \& Nissen, P. E. 1994, A\&A, 284, 37

Korn, A. J., Becker, S. R., Gummersbach, C. A., \& Wolf, B. 2000, A\&A, 353, 655

Korn, A. J., Keller, S. C., Kaufer, A., et al. 2002, A\&A, 385, 143

Kurt, C. M., Dufour, R. J., \& Garnett, D. R., et al. 1999, ApJ, 518, 246

Kurucz, R. 1991, in Precision Photometry: Astrophysics of the Galaxy, ed. A. G. Philip, A. R. Upgren, \& P. L. Janes (Schenectady: L. Davis Press), 27

Lennon, D. J., Brown, P. J. F., \& Dufton, P. L. 1988, A\&A, 195, 208

Lennon, D. J., Dufton, P. L., \& Crowley, C. 2003, A\&A, 398, 455

Lennon, D. J., Dufton, P. L., Mazzali, P. A., Pasian, F., \& Marconi, G. 1996, A\&A, 314, 243

Luck, R. E., Moffett, T. J., Barnes, T. G., \& Gieren, W. P. 1998, ApJ, 115,605

Mooney, C. J., Rolleston, W. R. J., Keenan, F. P., et al. 2001, MNRAS, 326,1101

Pagel, B. E. J. 1992, in New Aspects of Magellanic Cloud Research, ed. G. Klare (Springer), 330

Pagel, B. E. J., \& Tautvaišienè, G. 1998, MNRAS, 299, 535

Peimbert, M., Peimbert, A., \& Ruiz, M. T. 2000, ApJ, 541, 688

Peimbert, M., Torres-Peimbert, S., \& Ruiz, M. T. 1992, RMXAA, 24, 155

Reyes, C. 1999, IAU Symp., 190, 282

Rolleston, W. R. J. 1991, Ir. Astron. J., 20, 60

Rolleston, W. R. J., Brown, P. J. F., Dufton, P. L., \& Howarth, I. D. 1996, A\&A, 315, 95

Rolleston, W. R. J., Dufton, P. L., Fitzsimmons, A., Howarth, I. D., \& Irwin, M. J. 1993, A\&A, 277, 10
Rolleston, W. R. J., Dufton, P. L., McErlean, N. D., \& Venn, K. A. 1999a, A\&A, 348, 728

Rolleston, W. R. J., Hambly, N. C., Keenan, F. P., Dufton, P. L., \& Saffer, R. A. 1999b, A\&A, 347, 69

Rolleston, W. R. J., Smartt, S. J., Dufton, P. L., \& Ryans, R. S. I. 2000, A\&A, 363, 537

Russell, S. C., \& Bessell, M. S. 1989, ApJS, 70, 865

Russell, S. C., \& Dopita, M. A. 1990, ApJS, 74, 93

Russell, S. C., \& Dopita, M. A. 1992, ApJ, 384, 508

Schönberner, D., Herrero, A., Becker, S. R., et al. 1988, A\&A, 197, 209

Shaver, P. A., McGee, R. X., Newton, L. M., Danks, A. C., \& Pottasch, S. R. 1983, MNRAS, 204, 53

Spite, M., \& Spite, F. 1990, A\&A, 234, 67

Spite, M., Barbury, B., \& Spite, F. 1989, A\&A, 222, 35

Spite, F., Richtler, T., \& Spite, M. 1991, A\&A, 252, 557

Testor, G. 2001, A\&A, 372, 667

Venn, K. A. 1999a, ApJ, 518, 405

Venn, K. A. 1999b, IAU Symp., 190, 200

Venn, K. A., Brooks, A. M., Lambert, D. L., et al. 2002, ApJ, 565, 571

Vidal, C. R., Cooper, J., \& Smith, E. W. 1973, ApJS, 25, 37

Wayte, S. R. 1990, ApJ, 355, 473

Welty, D. E., Lauroesch, J. T., Blades, J. C., Hobbs, L. M., \& York, D. G. 1997, ApJ, 489, 672

Westerlund, B. 1997, in The Magellanic Clouds (Cambridge University Press) 